\title{
A GAUSSIAN APPROXIMATION OF THE DISTRIBUTED COMPUTING PROCESS
}

\author{
O. V. Lukashenko ${ }^{1}$, E. V. Morozov ${ }^{2}$, and M. Pagano ${ }^{3}$
}

\begin{abstract}
The authors propose a refinement of the stochastic model describing the dynamics of the Desktop Grid (DG) project with many hosts and many workunits to be performed, originally proposed by Morozov et al. in 2017. The target performance measure is the mean duration of the runtime of the project. To this end, the authors derive an asymptotic expression for the amount of the accumulated work to be done by means of limit theorems for superposed on-off sources that lead to a Gaussian approximation. In more detail, depending on the distribution of active and idle periods, Brownian or fractional Brownian processes are obtained. The authors present the analytic results related to the hitting time of the considered processes (including the case in which the overall amount of work is only known in a probabilistic way), and highlight how the runtime tail distribution could be estimated by simulation. Taking advantage of the properties of Gaussian processes and the Conditional Monte-Carlo (CMC) approach, the authors present a theoretical framework for evaluating the runtime tail distribution.
\end{abstract}

Keywords: Gaussian approximation; distributed computing; fractional Brownian motion

DOI: $10.14357 / 19922264190215$

\section{Introduction}

Gaussian processes are widely used in the performance analysis of telecommunication systems for their analytic tractability and arguments based on the central-limit theorem that make them suitable in case of a large number of independent contributions. For instance, these models are able to capture, in a simple and parsimonious way, the properties of self-similarity and long-range dependence, inherent to multimedia network traffic [1,2]. These properties dramatically increase the difficulty of the probabilistic analysis and, as a consequence, in many cases only Monte-Carlo simulation can be used. The fractional Brownian motion (FBM) is one of the most studied self-similar long-range dependent Gaussian processes due to its simplicity. Its use as traffic model is supported by the following theoretical analysis [3]: the sum of an increasing number of the so-called on-off inputs, with either on-times or off-times having a heavy-tailed distribution with infinite variance, converges weakly to an FBM, after an appropriate time scaling.

In this paper, the applicability of FBM for highperformance computing is considered. In that framework, computing clusters and computational Grid systems are the main tools: computing clusters are based on computing nodes connected by a high-speed network, while computational Grid systems include geographically dispersed computing nodes connected by a relatively slow network.

Desktop Grid belongs to the latter class. The DG combines nondedicated hosts (typically, desktops/laptops owned by volunteers) over the Internet to process loosely coupled workunits (computational tasks). Desktop Grids utilize the idle host resources, providing potentially huge, although highly variable, computing power. (For example, the DG project EinsteinHOME aggregates peak performance at about 1 PetaFLOPS [4].) Typically, DGs are managed by a scientific community that utilizes the resources to complete a $D G$ project which consists of a (usually finite) number of workunits. Thus, the runtime of the DG project is the time to complete all the workunits and it is desirable to minimize it.

Minimization of the DG project runtime may be performed by means of scheduling optimization [5-7]. Additional information on the hosts, such as reliability and availability, can be used to improve the efficiency of DGs $[8,9]$, In $[10,11]$, the focus is placed on the so-called workunit replication mechanism for reliability purposes. However, to the best of our knowledge, the estimation of the runtime of a DG project remains generally an unsolved issue, and it is the main motivation of this paper.

\footnotetext{
${ }^{1}$ Institute of Applied Mathematical Research of Karelian Research Centre of RAS, 11 Pushkinskaya Str., Petrozavodsk 185910, Republic of Karelia, Russian Federation; Petrozavodsk State University, 33 Lenin Str., Petrozavodsk 185910, Republic of Karelia, Russian Federation, lukashenko@krc.karelia.ru

${ }^{2}$ Institute of Applied Mathematical Research of Karelian Research Centre of RAS, 11 Pushkinskaya Str., Petrozavodsk 185910, Republic of Karelia, Russian Federation; Petrozavodsk State University, 33 Lenin Str., Petrozavodsk 185910, Republic of Karelia, Russian Federation, emorozov@karelia.ru

${ }^{3}$ University of Pisa, 43 Lungarno Pacinotti, Pisa 56126, Italy, m.pagano@iet.unipi.it
} 
Desktop Grids have several important distinctive features when compared to computational Grids or computing clusters. First of all, hosts, being nondedicated, possess individual availability periods. Moreover, the management server of a DG is not able to obtain information on the current state of the hosts (such as "computing," "suspended," etc.). These two issues make the estimation of the runtime of a DG project a hard problem.

The execution of a DG project can be divided into two stages. During the first phase, the number of workunits is greater than the number of hosts and, thus, each host will receive at least one workunit. In the second stage, all the available workunits are dispatched and there are available (idle) hosts. In this paper, the focus is on the duration of the first phase which is studied by means of a Gaussian approximation of the overall work. The study of the second stage requires a completely different probabilistic technique, which relies on the theory of order statistics and the asymptotic properties of renewal processes, and is postponed for a future work. Thus, in what follows, runtime will relate to the first stage of the project solely.

We describe the availability patterns of the hosts by treating each of them as an individual on-off source which processes workunits during on periods. Our approach is based on the asymptotics of the (properly scaled) superposition of a large number of independent on-off sources. It is well-known [3] that after an appropriate scaling, the limiting process describing the summary workload in the system turns out to be Brownian motion (BM), when the sojourn times are light-tailed, while it becomes fractional Brownian motion in case of heavy-tailed sojourn times. Then, the problem reduces to the calculation of the hitting time of the given threshold $D$ by the process of accumulated work which is a well-known topic in probability theory.

The paper is organized as follows. Section 2 presents the theoretical background related to FBM, including functional limit theorems for the cumulative work performed by an increasing number of on-off sources. Then, Section 3 describes the model and summarizes the available analytic results, while Section 4 is devoted to the evaluation of the runtime tail distribution by means of the CMC method which potentially leads to variance reduction of the estimate of the runtime. Finally, in Section 5, the main contributions of the paper are presented and some future research issues are discussed.

\section{Theoretical Background}

In this section, let us recall the basic definitions about FBM and how it is related to the limiting theorems for the superposition of independent on-off sources.

\subsection{Fractional Brownian motion}

The FBM $\left\{B_{H}(t), t \in \mathbb{R}\right\}$ is a Gaussian centered process with $B_{H}(0)=0$, stationary increments, and the following covariance function:

$$
\begin{aligned}
K_{H}(t, s) & :=\mathbb{E}\left[B_{H}(t) B_{H}(s)\right] \\
& =\frac{1}{2}\left[|t|^{2 H}-|t-s|^{2 H}+|s|^{2 H}\right], s, t \geq 0
\end{aligned}
$$

where $H \in(0,1)$ is the so-called Hurst parameter. It is easy to verify that $B_{H}(t)$ is a self-similar process with self-similarity parameter $H$, i. e., for each $c>0$,

$$
c^{-H} B_{H}(c t) \stackrel{d}{=} B_{H}(t)
$$

where $\stackrel{d}{=}$ denotes equality in distribution.

Fractional Brownian motion is widely used for modeling purposes due to its Gaussianity (that typically arises under aggregation conditions) and parsimonious description (apart from mean and variance, its behavior is unambiguously determined by $H$ ).

When $H>1 / 2$, FBM is a long-range dependent process since the autocorrelation of the corresponding increment process is nonsummable. For more details on FBM and its properties, see [12].

\subsection{Limit theorems for distributed computing processes}

Let us assume that the DG consists of $N$ heterogeneous hosts which can be considered as independent on-off sources. In more detail, let us suppose that there are $n$ types of hosts $(n<N)$ and denote by $N_{i}$ the number of $i$-type hosts, i. e., $\sum_{i=1}^{n} N_{i}=N$. Moreover, let $R_{i}$ denote the amount of processed work per unit time for $i$-type hosts and let $\left\{I^{(i)}(t), t \geq 0\right\}$,

$$
I^{(i)}(t)= \begin{cases}R_{i}, & t \in \text { on-period } \\ 0, & t \in \text { off-period }\end{cases}
$$

be the on-off process that characterizes the activity/silent periods of the corresponding hosts (Fig. 1). For sake of simplicity, it is assumed that for each host, both on and off periods are sequences of i.i.d. (independent and identically distributed) random variables (RVs) and mutually independent. Moreover, as already stated, the

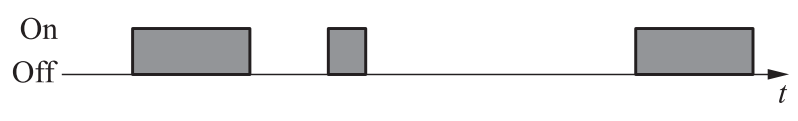

Figure 1 On-off model 
on-off processes modeling the contribution of different hosts are assumed to be independent.

The cumulative processed work, i.e., the aggregated amount of work provided by all $N$ hosts, during the time interval $[0, t]$ is given by

$$
A(t)=\int_{0}^{t}\left(\sum_{i=1}^{n} \sum_{k=1}^{N_{i}} I_{k}^{(i)}(u)\right) d u
$$

where $I_{k}^{(i)}$ are the independent copies of $I^{(i)}, i=1, \ldots$ $\ldots, n$. Moreover, for the $i$-type $(i=1, \ldots, n)$ hosts, let us denote by $\mu_{\text {on }}^{i}, \sigma_{\text {on }}^{i}, \mu_{\text {off }}^{i}$, and $\sigma_{\text {off }}^{i}$ the mean length and standard deviation (that may be infinite) of the duration of the on and off periods, respectively.

The statistical behavior of $A(t)$ is determined by the distributions $F_{\text {on }}^{i}$ and $F_{\text {off }}^{i}$ of the on and off periods for each type of hosts, namely, by their tail. In more detail, in case of infinite variance, let us assume that as $x \rightarrow \infty$,

$$
\begin{aligned}
& 1-F_{\mathrm{on}}^{i}(x) \sim \ell_{\mathrm{on}}^{i} x^{-\alpha_{\mathrm{on}}^{i}} L_{\mathrm{on}}^{i}(x) ; \\
& 1-F_{\mathrm{off}}^{i}(x) \sim \ell_{\mathrm{off}}^{i} x^{-\alpha_{\mathrm{off}}^{i}} L_{\mathrm{off}}^{i}(x)
\end{aligned}
$$

where $a \sim b$ means that $a / b \rightarrow 1 ; \ell_{\text {on }}^{i}$ and $\ell_{\text {off }}^{i}$ are the positive constants; the exponents $\alpha_{\text {on }}^{i}$ and $\alpha_{\text {off }}^{i} \in(1,2)$; and the functions $L_{\text {on }}^{i}$ and $L_{\text {off }}^{i}$ are slowly varying at infinity, i. e., for any $t>0$,

$$
\lim _{x \rightarrow \infty} \frac{L^{i}(t x)}{L^{i}(x)}=1, i=1, \ldots, n .
$$

Instead, if $\sigma_{\text {on }}^{i}$ and $\sigma_{\text {off }}^{i}<\infty$, we set $\alpha_{\text {on }}^{i}=\alpha_{\text {off }}^{i}=2$.

It has been shown in [3] that the scaled process of cumulative work arrived during interval $[0, T t]$ converges weakly to a sum of the i.i.d. FBM's, provided that

(1) $N_{i} \rightarrow \infty$ such that $\lim _{N \rightarrow \infty} N_{i} / N>0, i=1, \ldots$ $\ldots, n$; and

(2) the scaling factor $T \rightarrow \infty$.

This functional limit theorem leads to the following approximation:

$$
\begin{aligned}
A(t T) \approx T & \left(\sum_{i=1}^{n} R_{i} N_{i} \frac{\mu_{\mathrm{on}}^{i}}{\mu_{\mathrm{on}}^{i}+\mu_{\mathrm{off}}^{i}}\right) t \\
& +\sum_{i=1}^{n} T^{H_{i}} R_{i} \sqrt{L_{i}(T) N_{i}} c_{i} B_{H_{i}}(t)
\end{aligned}
$$

where $c_{i}$ are the positive constants; $L_{i}$ are the slowly varying at infinity functions (expressed in terms of the given parameters); and $B_{H_{i}}$ are the independent FBMs with the Hurst parameters $H_{i}$ given by

$$
H_{i}=\frac{3-\min \left(\alpha_{\mathrm{on}}^{i}, \alpha_{\mathrm{off}}^{i}\right)}{2} \in\left(\frac{1}{2}, 1\right), i=1, \ldots, n \text {. }
$$

Thus, the cumulative work processed by a large number of independent hosts (with heavy-tailed distributions of the on-off periods) is approximated by a superposition of independent FBMs $\left\{B_{H_{i}}(t)\right\}, i=1, \ldots, n$, with a linear drift that depends on the rates $R_{i}$ and the average duty cycle.

Instead, if for all types of hosts the variances of the sojourn times are finite (i. e., $\sigma_{\text {on }}^{i}, \sigma_{\text {off }}^{i}<\infty \forall i=1, \ldots, n$ ), then the limiting (scaled) process becomes

$$
T\left(\sum_{i=1}^{n} \frac{R_{i} N_{i} \mu_{\mathrm{on}}^{i}}{\mu_{\mathrm{on}}^{i}+\mu_{\mathrm{off}}^{i}}\right) t+\left(\sqrt{T} \sum_{i=1}^{n} R_{i} \sqrt{N_{i}} c_{i}\right) W(t)
$$

where $W(t)$ is the Wiener process, and the constants $c_{i}$ are given by

$$
c_{i}=\sqrt{\frac{\left(\mu_{\mathrm{off}}^{i} \sigma_{\mathrm{on}}^{i}\right)^{2}+\left(\mu_{\mathrm{on}}^{i} \sigma_{\mathrm{off}}^{i}\right)^{2}}{\left(\mu_{\mathrm{on}}^{i}+\mu_{\mathrm{off}}^{i}\right)^{3}}} .
$$

Finally, it is worth mentioning that taking the limits in reverse order, the (scaled) process of cumulative work converges to a Levy stable motion, an infinite variance process with stationary and independent increments [13]; however, such model is beyond the scope of this paper as in DG, the experimental data confirmed the convergence to processes with finite variance.

\section{Model Description and Performance Measures}

The above functional limit theorems provide a theoretical motivation to consider the following model for the cumulative processed work:

$$
A(t)=m t+X(t)
$$

where $X$ is the centered Gaussian process with stationary increments (FBM or the sum of independent FBM, in case of heterogeneous systems), which describes random fluctuations around the linearly increasing mean. Such type of stochastic process was previously suggested as the model of network traffic (see [14] for more details).

Let us denote by $\tau_{D}$ the runtime of the DG project where $D$ denotes the required amount of work. Thus, $\tau_{D}$ represents the hitting time of the process $\{A(t)\}$ :

$$
\tau_{D}=\min \{t: A(t) \geq D\},
$$

i. e., the first time the process $\{A(t)\}$ hits the threshold $D$. Then, the original problem is reduced to the calculation (or estimation) of some useful performance characteristics, such as the mean hitting time. 


\subsection{Available analytic results}

Let us recall the available analytic results for different types of Gaussian processes, corresponding to the different limiting cases.

\subsubsection{Wiener case}

When $X$ is a Wiener process (i.e., $X=\sigma B_{1 / 2}$ ), the density of $\tau_{D}$ is available in explicit form [15]:

$$
\begin{array}{r}
\mathbb{P}\left(\tau_{D} \in d t\right)=\frac{D}{\sqrt{2 \pi} \sigma t^{3 / 2}} \exp \left(-\frac{(D-m t)^{2}}{2 \sigma^{2} t}\right) d t \\
=: f_{\tau}(t \mid D) d t
\end{array}
$$

In this case, the corresponding expected value $\mathbb{E}\left[\tau_{D}\right]$ is the ratio between the given amount of the work $D$ and the mean processing rate [15]:

$$
\mathbb{E}\left[\tau_{D}\right]=\frac{D}{m} .
$$

\subsubsection{Fractional Brownian motion case}

When the limiting process is an FBM, only asymptotic results and some bounds for the distribution of $\tau_{D}$ are available.

In [16], the following bounds (quite inaccurate when $H$ is close to 1 , see Fig. 2) for the moments of the hitting time were obtained for $1 / 2 \leq H<1$ :

$$
\begin{aligned}
& \frac{1}{\sqrt{2 \pi}}\left(\frac{2 H D}{n-H} L_{n}(D, H, m)\right. \\
& \left.-\frac{(2 H-1) m}{n+1-H} L_{n+1}(D, H, m)\right) \leq \mathbb{E}\left[\tau_{D}^{n}\right] \\
& \leq \frac{1}{\sqrt{2 \pi}}\left(\frac{H D}{n-H} L_{n}(D, H, m)\right. \\
& \left.+\frac{(1-H) m}{n+1-H} L_{n+1}(D, H, m)\right)
\end{aligned}
$$

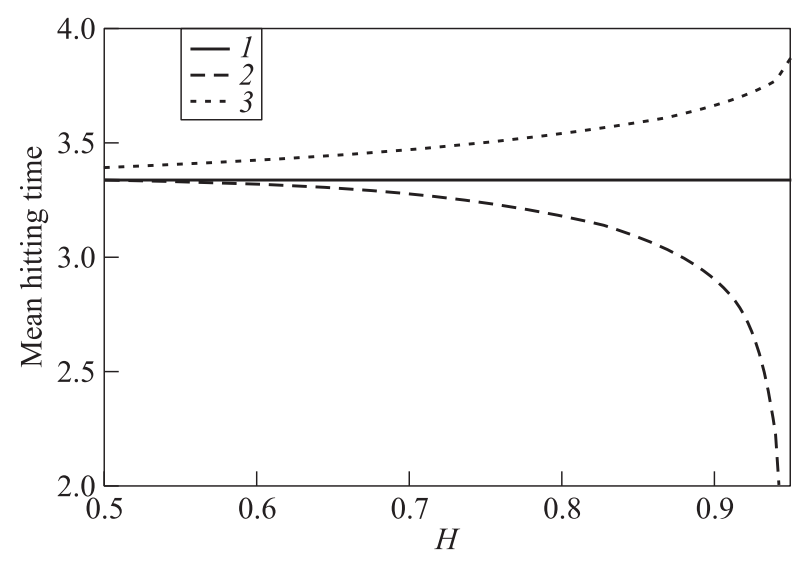

Figure 2 Bounds for the mean hitting time $(D=10, m=3)$ : $1-D / m ; 2-$ lower bound; and 3 - upper bound where

$$
\begin{aligned}
& L_{n}(D, H, m) \\
= & \int_{0}^{\infty} \exp \left\{-\frac{1}{2}\left(D t^{-H /(n-H)}-m t^{(1-H) /(n-H)}\right)^{2}\right\} d t .
\end{aligned}
$$

Additionally, the following asymptotic was derived for the large values of level $D$ :

$$
\lim _{D \rightarrow \infty} \frac{\mathbb{E}\left[\tau_{D}^{n}\right]}{D^{n}}=m^{-n}
$$

for all $n \geq 1, m>0$, from which it is quite straightforward to show that for all $n \geq 1$,

$$
\frac{\tau_{D}}{D} \stackrel{L_{n}}{\longrightarrow} \frac{1}{m} \text { as } D \rightarrow \infty
$$

where $\stackrel{L_{n}}{\longrightarrow}$ means convergence in $L_{n}$ space.

\subsubsection{General case}

In the general case, to derive asymptotic (for large values of $D$ ) for the distribution of $\tau_{D}$, it is possible to take advantage of the following identity:

$$
\mathbb{P}\left(\tau_{D} \leq T\right)=\mathbb{P}\left(\sup _{t \in[0, T]} A(t) \geq D\right) .
$$

The distribution of the maximum of Gaussian processes over a finite interval is a well-studied problem. In more detail, for any Gaussian process with stationary increments and strictly monotonically increasing and convex variance such that $\lim _{t \rightarrow 0} \operatorname{Var}(X(t)) / t=0$, the following asymptotic holds [17]:

$$
\mathbb{P}\left(\sup _{t \in[0, T]} A(t) \geq D\right) \sim \Phi\left(\frac{D-m T}{\sqrt{\operatorname{Var}(X(T))}}\right)
$$

$$
\text { as } D \rightarrow \infty
$$

where $\Phi$ denotes the tail distribution of the standard normal RV $N(0,1)$.

\subsection{A possible generalization}

It seems quite natural to consider the setting in which the threshold $D$ is an $\mathrm{RV}$ which is independent of the process $X$ in (1). Such a setting seems to be highly motivated by practice because it is more realistic that the exact value of the quantity $D$ is not available, and it is known in part. This incomplete information can be reflected by introducing the probability density function (PDF) $f_{D}$ of $D$, which is assumed to be predefined. Provided that $X$ in (1) is a Wiener process and, hence, 
the conditional density $f_{\tau}(t \mid D)$ in (2) is known, one can write the density of the $\mathrm{RV} \tau_{D}$ as

$$
f_{\tau}(x)=\int_{y=0}^{\infty} f_{\tau}(x \mid y) f_{D}(y) d y .
$$

In general, one can calculate this density only by numerical methods but for some cases, it is possible to derive its expression in terms of special functions. For example, when $D$ is exponential with parameter $\lambda$, one can obtain the following expression:

$$
\begin{array}{r}
f_{\tau}(x)=\frac{\lambda}{\sqrt{2 \pi} \sigma x^{3 / 2}} \exp \left(-\frac{m^{2} x}{2 \sigma^{2}}+\frac{\gamma^{2}}{8 \beta(x)}\right) \\
\quad \times(2 \beta(x))^{-1 / 2} D_{-1}\left(\frac{\gamma}{\sqrt{2 \beta(x)}}\right)
\end{array}
$$

where

$$
\gamma=\lambda-\frac{m}{\sigma^{2}} ; \quad \beta(x)=\frac{1}{2 \sigma^{2} x} ;
$$

and $D_{p}$, Re $p<0$, is the parabolic cylinder function [18]. Numeric calculation of the expression (3) is shown in Fig. 3.

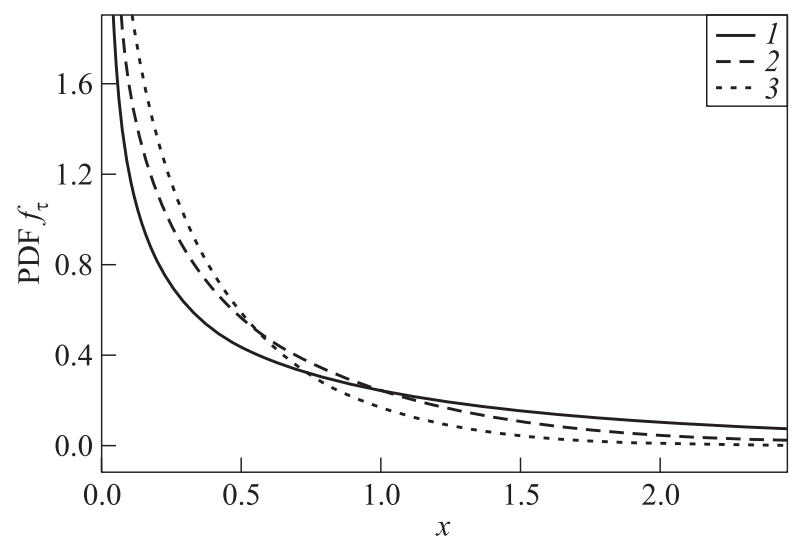

Figure 3 Probability density function of $\tau_{D}$ for different values of $m(\lambda=1): 1-m=1 ; 2-2$; and $3-m=3$

\section{Estimation via Monte Carlo}

A more flexible alternative to analytic results is represented by simulation that in our case can be used to estimate

$$
\pi(T):=\mathbb{P}\left(\tau_{D}>T\right) .
$$

Such probability could be extremely small for large values of $T$; thus, its estimation with a given accuracy requires to generate a large number of sample paths of the process $X$. However, for such type of rare events, it is possible to apply a special case of the well-known $\mathrm{CMC}$ method which always leads to variance reduction.
The method, originally proposed by some of the authors in [19-21] and named Bridge Monte Carlo (BMC), is based on the idea of expressing the target probability as the expectation of a function of the Bridge $Y:=\left\{Y_{t}\right\}$ of the Gaussian process $X$, i. e., the process obtained by conditioning $X$ to reach a certain level at some prefixed time instant $\tau$ :

$$
Y(t)=X(t)-\psi(t) X(\tau)
$$

where $\psi$ can be easily expressed in terms of the the covariance function $\Gamma(s, t)$ of the process $X$

$$
\psi(t):=\frac{\Gamma(t, \tau)}{\Gamma(\tau, \tau)} .
$$

Since the variance of $X$ is an increasing function of $t$ in all models we consider, it is easy to see that $\psi(t)>0$ for all $t \geq 0$. Moreover, for any $t, Y(t)$ is independent of $X(\tau)$ since

$$
\mathbb{E}[X(\tau) Y(t)]=\Gamma(\tau, t)-\frac{\Gamma(t, \tau)}{\Gamma(\tau, \tau)} \Gamma(\tau, \tau)=0
$$

and $(X(\tau), Y(t))$ has bivariate normal distribution.

Let $\mathbb{T}=[0, T]$, then the target probability can be expressed in the following way:

$$
\begin{array}{r}
\pi(T)=\mathbb{P}\left(\sup _{t \in[0, T]} A(t) \geq D\right) \\
=\mathbb{P}(\forall t \in \mathbb{T}: m t+X(t) \leq D) \\
=\mathbb{P}\left(\forall t \in \mathbb{T}: X(\tau) \leq \frac{D-Y(t)-m t}{\psi(t)}\right) \\
=\mathbb{P}\left(X(\tau) \leq \inf _{t \in T} \frac{D-Y(t)-m t}{\psi(t)}\right) \\
=\mathbb{P}(X(\tau) \leq \bar{Y})
\end{array}
$$

where

$$
\bar{Y}:=\inf _{t \in \mathbb{T}} \frac{D-Y(t)-m t}{\psi(t)} .
$$

Finally, the considered probability can be rewritten as follows:

$$
\pi(T)=\mathbb{P}\left(X_{\tau} \leq \bar{Y}\right)=\mathbb{E}\left[\Psi\left(\frac{\bar{Y}}{\sqrt{\Gamma(\tau, \tau)}}\right)\right]
$$

where independence between $\bar{Y}$ and $X_{\tau}$ is used and $\Psi$ denotes the cumulative distribution function of a standard normal variable.

Hence, given $N$ samples $\left\{\bar{Y}^{(n)}, n=1, \ldots, N\right\}$ of $\bar{Y}$, the estimator of $\pi(T)$ is defined as follows:

$$
\widehat{\pi}_{N}^{\mathrm{BMC}}:=\frac{1}{N} \sum_{n=1}^{N} \Psi\left(\frac{\bar{Y}^{(n)}}{\sqrt{\Gamma(\tau, \tau)}}\right) .
$$


Note that

$$
\Psi\left(\frac{\bar{Y}}{\sqrt{\Gamma(\tau, \tau)}}\right)=\mathbb{E}[I(X(\tau) \leq \bar{Y}) \mid \bar{Y}]
$$

and, therefore, the BMC approach is actually a special case of the CMC method; so, one can expect that the $\mathrm{BMC}$ estimator implies variance reduction (with regard to crude Monte-Carlo simulation) in the estimation of the target probability $\pi(T)$ as also justified by the previous experience when such a method was successfully applied for estimation some other rare-event probabilities related to Gaussian processes [22].

\section{Concluding Remarks and Future Research}

In this paper, a stochastic model describing the dynamics of a DG project with many hosts and many workunits to be performed, originally proposed in [23], is presented. It is assumed that the project can be described by the socalled on-off model where the hosts are on-off sources of the workunits and the basic process is the completed work. It is assumed that the hosts' working sessions can have both light- and heavy-tailed distributions. Then, an approximation of the basic process, based on the asymptotics of the superposed on-off sources, is applied. The suggested approach leads to a Gaussian approximation of the process of the completed work. Finally, a simulation framework for the evaluation of the runtime of the project, using the properties of Gaussian processes and CMC simulation, is presented.

Although this note is focused on estimation of the runtime related to the 1st stage of the project completion when the number of workunits is bigger than the number of hosts, the 2 nd stage could also be relevant. In more detail, it can be considered as a collection of the "tails" of the workunit remaining times. From this point of view, the completion time of the 2nd stage of the project can be interpreted as the longest remaining time and analyzed by means of the asymptotic results of renewal theory. Moreover, since the workunits are assumed to be independent, to evaluate the duration of the 2 nd stage, it seems promising to apply the theory of order statistics and interpret the completion time as the maximal order statistics.

\section{Acknowledgments}

The study was carried out under state order to the Karelian Research Centre of the Russian Academy of Sciences (Institute of Applied Mathematical Research KarRC RAS) and supported by the Russian Foundation for Basic Research, projects 18-07-00187, 18-07-00147, 18-07-00156, and 19-07-00303.

\section{References}

1. Leland, W. E., M. S. Taqqu, W. Willinger., and D. V. Wilson. 1994. On the self-similar nature of ethernet traffic (extended version). IEEE ACM T. Network. 2(1):1-15.

2. Willinger, W., M. S. Taqqu, W. E. Leland, and D. Wilson. 1995. Self-similarity in high-speed packet traffic: Analysis and modeling of Ethernet traffic measurements. Stat. Sci. 10(1):67-85.

3. Taqqu, M. S., W. Willinger, and R. Sherman. 1997. Proof of a fundamental result in self-similar traffic modeling. Comp. Comm. R. 27:5-23.

4. BOINCstats. 2017. Available at: https://boincstats.com (accessed May 7, 2019).

5. Kondo, D., D. P. Anderson, and J. McLeod VII. 2007. Performance evaluation of scheduling policies for volunteer computing. 3rd IEEE Conference (International) on $e$-Science and Grid Computing Proceedings. IEEE. 221227.

6. Estrada, T., and M. Taufer. 2012. Challenges in designing scheduling policies in volunteer computing. Desktop grid computing. Eds C. Cérin and G. Fedak. CRC Press. 167-190.

7. Durrani, N., and J. Shamsi. 2014. Volunteer computing: Requirements, challenges, and solutions. J. Netw. Comput. Appl. 39:369-380.

8. Sonnek, J., M. Nathan, A. Chandra, and J. Weissman. 2006. Reputation-based scheduling on unreliable distributed infrastructures in distributed computing systems. 26th IEEE Conference (International) on Distributed Computing Systems Proceedings. IEEE. Art. No. 30. P. 1-8.

9. Watanabe, K., M. Fukushi, and M. Kameyama. 2011. Adaptive group-based job scheduling for high performance and reliable volunteer computing. J. Information Processing 19:39-51.

10. Xavier, E., R. Peixoto, and J. da Silveira. 2013. Scheduling with task replication on desktop grids: Theoretical and experimental analysis. J. Comb. Optim. 30(3):520-544.

11. Chernov, I. A., and N. N. Nikitina. 2015. Virtual screening in a Desktop Grid: Replication and the optimal quorum. Parallel computing technologies. Ed. V. Malyshkin. Lecture notes in computer science ser. Springer. 9251:258-267.

12. Samorodnitsky, G., and M. S. Taqqu. 1994. Stable nonGaussian random processes: Stochastic models with infinite variance. Chapman \& Hall. $632 \mathrm{p}$.

13. Mikosch, T., S. Resnick, H. Rootzén, and A. Stegeman. 2002. Is network traffic approximated by stable Levy motion or fractional Brownian motion? Ann. Appl. Probab. 12(1):23-68.

14. Norros, I. 1994. A storage model with self-similar input. Queueing Syst. 16:387-396.

15. Borodin, A. N., and P. Salminen. 2002. Handbook of Brownian motion - facts and formulae. Birkhäuser. 685 p. 
16. Michna, Z. 1999. On tail probabilities and first passage times for fractional Brownian motion. Math. Method. Oper. Res. 49(2):335-354.

17. Caglar, M., and C. Vardar. 2013. Distribution of maximum loss of fractional Brownian motion with drift. Stat. Probabil. Lett. 83:2729-2734.

18. Gradshtein, I. S., I. M. Ryzhik, and A. Jeffrey, eds. 2015. Table of integrals, series and products. 8th ed. San Diego, CA: Academic Press. 1220 p.

19. Giordano, S., M. Gubinelli, and M. Pagano. 2005. Bridge Monte-Carlo: A novel approach to rare events of Gaussian processes. 5th St. Petersburg Workshop on Simulation Proceedings. St. Petersburg: St. Petersburg State University. 281-286.

20. Giordano, S., M. Gubinelli, and M. Pagano. 2007. Rare events of Gaussian processes: A performance compari- son between bridge Monte-Carlo and importance sampling. Next generation teletraffic and wired/wireless advanced networking. Eds. Y. Koucheryavy, J. Harju, and A. Sayenko. Lecture notes in computer science ser. Springer. 4712:269-280.

21. Lukashenko, O. V., E. V. Morozov, and M. Pagano. 2012. Performance analysis of Bridge Monte-Carlo estimator. Transactions of KarRC RAS 5:54-60.

22. Lukashenko, O. V., E. V. Morozov, and M. Pagano. 2017. On the efficiency of bridge Monte-Carlo estimator. Informatika i ee Primeneniya - Inform. Appl. 11(2):16-24.

23. Morozov, E., O. Lukashenko, A. Rumyantsev, and E. Ivashko. 2017. A Gaussian approximation of runtime estimation in a desktop grid project. 9th Congress (International) on Ultra Modern Telecommunications and Control Systems and Workshops. IEEE. 107-111.

Received April 15, 2019

\title{
Contributors
}

Lukashenko Oleg V. (b. 1986) - Candidate of Science (PhD) in physics and mathematics, scientist, Institute of Applied Mathematical Research of Karelian Research Centre of the Russian Academy of Sciences, 11 Pushkinskaya Str., Petrozavodsk 185910, Republic of Karelia, Russian Federation; associate professor, Petrozavodsk State University, 33 Lenin Str., Petrozavodsk 185910, Republic of Karelia, Russian Federation; lukashenko@krc.karelia.ru

Morozov Evsei V. (b. 1947) - Doctor of Science in physics and mathematics, professor, leading scientist, Institute of Applied Mathematical Research of Karelian Research Centre of the Russian Academy of Sciences, 11 Pushkinskaya Str., Petrozavodsk 185910, Republic of Karelia, Russian Federation; professor, Petrozavodsk State University, 33 Lenin Str., Petrozavodsk 185910, Republic of Karelia, Russian Federation; emorozov@karelia.ru

Pagano Michele (b. 1968) - PhD in Information Engineering, associate professor, University of Pisa, 43 Lungarno Pacinotti, Pisa 56126, Italy; m.pagano@iet.unipi.it

\section{ГАУССОВСКАЯ АППРОКСИМАЦИЯ ПРОЦЕССА РАСПРЕДЕЛЕННЫХ ВЫЧИСЛЕНИЙ*}

\author{
О. В. Лукашенко ${ }^{1,2}$, Е. В. Морозов ${ }^{1,2}$, М. Пагано ${ }^{3}$
}

${ }^{1}$ Институт прикладных математических исследований Карельского научного центра Российской академии наук

${ }^{2}$ Петрозаводский государственный университет

${ }^{3}$ Университет г. Пиза, Италия

\begin{abstract}
Аннотация: Продолжено изучение стохастической модели процесса динамики выполнения задачи в системе Desktop Grid при наличии многих пользователей, предложенной в 2017 г. Морозовым с соавт. Требуемой характеристикой выступает средняя продолжительность времени выполнения проекта. Гауссовская аппроксимация искомого процесса производится на основе предельных теорем для суперпозиции опoff источников. Приведен обзор известных аналитических результатов для требуемой характеристики, включая результаты для броуновского и дробного броуновского движения. Также показывается, как с помощью условного метода Монте-Карло оценить хвост распределения времени выполнения проекта.
\end{abstract}

Ключевые слова: гауссовская аппроксимация; распределенные вычисления; дробное броуновское движение

DOI: $10.14357 / 19922264190215$

\footnotetext{
* Финансовое обеспечение исследований осуществлялось из средств федерального бюджета на выполнение государственного задания КарНЦ РАН (Институт прикладных математических исследований КарНЦ РАН) и при финансовой поддержке РФФИ (проекты 18-07-00187, 18-07-00147, 18-07-00156 и 19-07-00303).
} 


\section{Литература}

1. Leland W.E., Taqqu M.S., Willinger W., Wilson D.V. On the self-similar nature of Ethernet traffic (extended version) // IEEE ACM T. Network., 1994. Vol. 2. Iss. 1. P. $1-15$.

2. Willinger W., Taqqu M.S., Leland W.E., Wilson D. Selfsimilarity in high-speed packet traffic: Analysis and modeling of Ethernet traffic measurements // Stat. Sci., 1995. Vol. 10. Iss. 1. P. 67-85.

3. Taqqu M.S., Willinger W., Sherman R. Proof of a fundamental result in self-similar traffic modeling // Comp. Comm. R., 1997. Vol. 27. P. 5-23.

4. BOINCstats, 2017. https://boincstats.com.

5. Kondo D., Anderson D.P., McLeod VII J. Performance evaluation of scheduling policies for volunteer computing // 3rd IEEE Conference (International) on e-Science and Grid Computing Proceedings. - IEEE, 2007. P. 221227.

6. Estrada T., Taufer M. Challenges in designing scheduling policies in volunteer computing // Desktop grid computing / Eds. C. Cérin, G. Fedak. - CRC Press, 2012. P. 167-190.

7. Durrani N., Shamsi J. Volunteer computing: Requirements, challenges, and solutions // J. Netw. Comput. Appl., 2014. Vol. 39. P. 369-380.

8. Sonnek J., Nathan M., Chandra A., Weissman J. Reputation-based scheduling on unreliable distributed infrastructures in distributed computing systems // 26th IEEE Conference (International) on Distributed Computing Systems Proceedings. - IEEE, 2006. Art. No. 30. P. $1-8$.

9. Watanabe K., Fukushi M., Kameyama M. Adaptive groupbased job scheduling for high performance and reliable volunteer computing // J. Information Processing, 2011. Vol. 19. P. 39-51.

10. Xavier E., Peixoto R., da Silveira J. Scheduling with task replication on desktop grids: Theoretical and experimental analysis // J. Comb. Optim., 2013. Vol. 30. Iss. 3. P. 520-544.

11. Chernov I. A., Nikitina N. N. Virtual screening in a desktop grid: Replication and the optimal quorum // Parallel computing technologies / Ed. V. Malyshkin. - Lecture notes in computer science ser. - Springer, 2015. Vol. 9251. P. 258-267.
12. Samorodnitsky G., Taqqu M.S. Stable non-Gaussian random processes: Stochastic models with infinite variance. Chapman \& Hall, 1994. 632 p.

13. Mikosch T., Resnick S., Rootzén H., Stegeman A. Is network traffic approximated by stable Levy motion or fractional Brownian motion? // Ann. Appl. Probab., 2002. Vol. 12. Iss. 1. P. 23-68.

14. Norros I. A storage model with self-similar input// Queueing Syst., 1994. Vol. 16. P. 387-396.

15. Borodin A. N., Salminen P. Handbook of Brownian motion - facts and formulae. - Birkhäuser, 2002. 685 p.

16. Michna $Z$. On tail probabilities and first passage times for fractional Brownian motion // Math. Method. Oper. Res., 1999. Vol. 49. Iss. 2. P. 335-354.

17. Caglar M., Vardar C. Distribution of maximum loss of fractional Brownian motion with drift // Stat. Probabil. Lett., 2013. Vol. 83. P. 2729-2734.

18. Table of integrals, series and products / Eds. I. S. Gradshtein, I. M. Ryzhik, A. Jeffrey. -8 ed. - San Diego, CA, USA: Academic Press, 2015. 1220 p.

19. Giordano S., Gubinelli M., Pagano M. Bridge Monte-Carlo: A novel approach to rare events of Gaussian processes // 5th St. Petersburg Workshop on Simulation Proceedings. - St. Petersburg: St. Petersburg State University, 2005. P. 281-286.

20. Giordano S., Gubinelli M., Pagano M. Rare events of Gaussian processes: A performance comparison between bridge Monte-Carlo and importance sampling // Next generation teletraffic and wired/wireless advanced networking / Eds. Y. Koucheryavy, J. Harju, A. Sayenko. Lecture notes in computer science ser. - Springer, 2007. Vol. 4712. P. 269-280.

21. Lukashenko O. V., Morozov E. V., Pagano M. Performance analysis of bridge Monte-Carlo estimator // Труды Карельского научного центра Российской академии наук, 2012. Т. 5. С. 54-60.

22. Lukashenko O. V., Morozov E. V., Pagano M. On the efficiency of bridge Monte-Carlo estimator // Информатика и её применения, 2017. Т. 11. Вып. 2. С. 16-24.

23. Morozov E., Lukashenko O., Rumyantsev A., Ivashko E. A Gaussian approximation of runtime estimation in a desktop grid project // 9th Congress (International) on Ultra Modern Telecommunications and Control Systems and Workshops. - IEEE, 2017. P. 107-111.

Поступила в редакцию 15.04.2019 